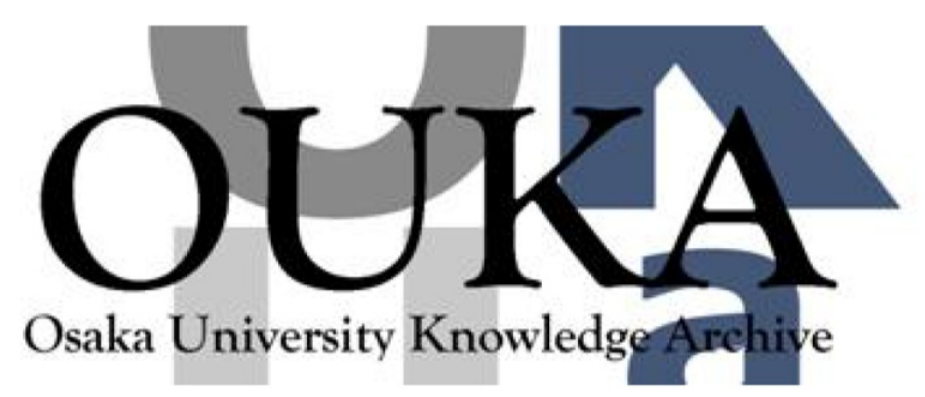

\begin{tabular}{|c|c|}
\hline Title & Defect imaging with guided waves in a pipe \\
\hline Author(s) & Hayashi, Takahiro; Murase, Morimasa \\
\hline Citation & $\begin{array}{l}\text { The Journal of the Acoustical Society of } \\
\text { America. } 117(4) \text { p. } 2134-\text { p. } 2140\end{array}$ \\
\hline Issue Date & $2005-04-08$ \\
\hline oaire:version & VoR \\
\hline URL & https://hdl. handle. net/11094/84488 \\
\hline rights & $\begin{array}{l}\text { Copyright } 2005 \text { Acoustical Society of America. } \\
\text { This article may be downloaded for personal use } \\
\text { only. Any other use requires prior permission } \\
\text { of the author and the Acoustical Society of } \\
\text { America. }\end{array}$ \\
\hline Note & \\
\hline
\end{tabular}

Osaka University Knowledge Archive : OUKA

https://ir. Library. osaka-u. ac. jp/

0saka University 


\title{
Defect imaging with guided waves in a pipe
}

\author{
Takahiro Hayashi ${ }^{\mathrm{a}}$ and Morimasa Murase \\ Faculty of Engineering, Nagoya Institute of Technology, Gokiso Showa Nagoya, 466-8555, Japan
}

(Received 22 September 2004; revised 6 January 2005; accepted 7 January 2005)

\begin{abstract}
Guided wave techniques are expected to become an effective means for rapid, long-range inspection of pipes. Such techniques still have many practical difficulties in application, however, due to the complex characteristics of guided waves such as dispersion and their multimodal nature. A defect imaging technique is developed in this study to overcome the complexities of guided wave inspection. Received signals are separated into single-mode waveforms with a mode extraction technique and then spatial waveforms on the pipe surface at an arbitrary time are reconstructed. The predicted waveforms can provide a defect image at the moment when an incident wave arrives at a defect region, which is based on a time-reversal technique. This defect imaging technique is experimentally verified using eight signals detected at eight different circumferential positions. Images of artificial defects are obtained with one-hole and two-hole test pipes, and increasing the frequency of incident waves increases the resolution of the images. Holes and pipe ends are recognizable in the images, but the reconstructed images contain some errors in the area behind the defects where guided waves do not propagate or do not reflect back to the receiving transducers. (C) 2005 Acoustical Society of America. [DOI: 10.1121/1.1862572]
\end{abstract}

PACS numbers: 43.60.Lq, 43.60.Fg, 43.20.Mv [YHB]

Pages: $2134-2140$

\section{INTRODUCTION}

A nondestructive evaluation (NDE) technique for pipework has been developed using guided waves acting as ultrasonic modes propagating longitudinally in a pipe. Guided wave NDE is expected to become a rapid long-range inspection technique ${ }^{1,2}$ in which an inspection tool emits guided waves from an accessible part of a pipe, then detects reflected or transmitted guided waves from damaged areas of the pipe that are inaccessible (such as buried or coated regions). Because guided waves propagate as forward or backward traveling waves, transducers lined up on the circumference of a pipe can fully detect reflected waves or transmitted waves. Reflected and transmitted waves contain all of the information about reflective walls and scattering objects. If the detected signals are processed properly, most of this information can be acquired. This characteristic of guided waves is highly beneficial for NDE when considering that not all energy due to refraction and scattering can be detected by NDE using bulk waves.

Guided waves in a pipe, however, have multimodal and dispersive characteristics similar to Lamb waves. ${ }^{3,4}$ These distinctive characteristics make it difficult to evaluate pipe damage and defects with sufficient accuracy and reliability using conventional pulse-echo and pitch-catch techniques to measure amplitude and velocity. For example, the phase and amplitude of signals received from a transducer will often be completely different even at one wavelength away in the longitudinal direction. This is because guided waves are a superposition of many modes having different wavelengths and wave structures. And, because these characteristics of guided waves depend on diameter, thickness, and the material properties of the pipe, the results obtained from one pipe test cannot be directly applied to other types of pipes.

${ }^{a)}$ Corresponding author; electronic mail: hayashi@nitech.ac.jp
Easy-to-use axisymmetric modes have therefore been adopted for guided wave inspections. Axisymmetric modes have a wide nondispersive region in the dispersion curves, particularly an axisymmetric torsional mode $\mathrm{T}(0,1)$ having constant phase and group velocities over all frequency regions. As such, incident pulse signals of axisymmetric modes maintain their waveforms and propagate long distances with a high signal-to-noise ratio. Also, single axisymmetric modes can be detected easily using axisymmetric transducers with no mixing of nonaxisymmetric modes.

However, because axisymmetric modes propagate no information concerning the circumferential direction (such as circumferential location, maldistribution, or size of defects) and defect characterization cannot be accomplished in an axisymmetric mode, several studies on nonaxisymmetric modes of guided waves in a pipe have been recently presented. Ditri ${ }^{5}$ investigated scattering waves from a circumferential crack using the $S$-parameter formalism and addressed the possibility of crack sizing. Lowe et al. ${ }^{6}$ and Alleyne $e t a l^{7}$ analyzed mode converted guided waves from part-circumferential notches in a pipe both experimentally, using the mode extraction technique, and theoretically, using a finite element model. $\mathrm{Li}$ and $\mathrm{Rose}^{8}$ experimentally verified the angular profile of guided waves that were estimated by normal mode expansion theory, and determined the position of transducers for defect detection. Demma et al. ${ }^{9}$ investigated the reflected guided waves from a part-circumferential notch when a torsional axisymmetric mode is emitted, both experimentally and through FE analysis.

In this paper we describe a defect imaging technique, in which nonaxisymmetric modes, as well as an axisymmetric mode, are considered and an image of defects is obtained after appropriate signal processing. Guided wave propagation at an arbitrary time and point on the surface of a pipe is predicted by a combination of the mode extraction technique 


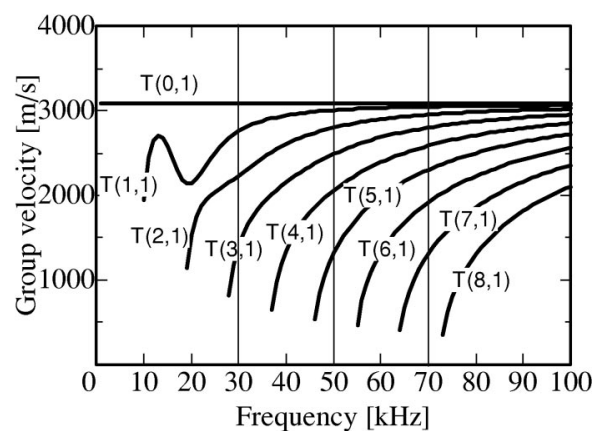

FIG. 1. Group velocity dispersion curves of torsional modes for an aluminum pipe with outer diameter $=111 \mathrm{~mm}$ and thickness $=3.5 \mathrm{~mm}$.

with the reconstruction of spatial waveforms. This technique is based on the time-reversed method proposed by Ing and Fink $^{3}$ in the sense that received waveforms are played back, but mode extraction and the reconstruction process are required.

The mode extraction technique, which is based on Lowe's method but includes negative circumferential orders, is described first, and defect imaging is described in detail. This imaging technique is then experimentally verified for one-hole and two-hole pipes at various frequencies.

\section{THEORY}

\section{A. Circumferential mode extraction}

The normal mode expansion technique gives the displacement on the surface of a pipe for a harmonic wave $\exp (-i \omega t)$ using the cylindrical coordinate system $(r, \theta, z)$ as

$$
u(\theta, z, t)=\sum_{n=-\infty}^{+\infty} \sum_{m=1}^{+\infty} \alpha_{n m} \exp \left(i n \theta+i k_{n m} z-i \omega t\right),
$$

where $n$ and $\alpha_{n m}$ represent the circumferential order number (family) and the amplitude of the $m$ th mode in the $n$th family, respectively. ${ }^{10-12}$ Wave numbers $k_{n m}$ consist of several propagating modes with real values and an infinite number of nonpropagating (evanescent) modes having complex values. $^{12}$

Torsional modes $\mathrm{T}(n, m)$ dominated mainly by a displacement in the circumferential direction are now considered. For additional details about indices $n$ and $m$, the reader should refer to Ref. 13, and to the dispersion curves of Fig. 1. Normally, guided wave NDE uses propagating modes with real wave numbers $k_{n m}$. For axisymmetric torsional modes $\mathrm{T}(0, m)$, the minimum frequencies above which the $m$ th propagating modes occur (the cutoff frequencies) are given for general-purpose pipes when the pipe wall thickness is small compared to the radius as

$$
f_{c}=\frac{c_{T}}{2 d}(m-1)
$$

where $c_{T}$ and $d$ are the transverse wave velocity and the thickness of the pipe, respectively. General-purpose pipes, such as schedule pipes, are at most approximately $15 \mathrm{~mm}$ thick with a transverse wave velocity of approximately 3200 $\mathrm{m} / \mathrm{s}$. The cutoff frequency for $m=2$ is therefore greater than $106 \mathrm{kHz}$ in Eq. (2). When we consider the frequency range from 20 to $100 \mathrm{kHz}$, used widely in long-range pipe inspections, modes greater than $m=2$ can be neglected. The displacement on the surface of a pipe in Eq. (1) can be rewritten as

$$
u(\theta, z, t)=\sum_{n=-\infty}^{+\infty} \alpha_{n} \exp \left(i n \theta+i k_{n} z-i \omega t\right),
$$

where $\alpha_{n 1} \equiv \alpha_{n}$ and $k_{n 1} \equiv k_{n}$, in order to simplify the representation.

Signals received in a small section $r_{0} d \theta$ at $z=z_{R}$ and $\theta=\theta$ are given by $u\left(\theta, z_{R}, t\right) r_{0} d \theta$. Multiplying a weight function $\exp \left(-i n_{E} \theta\right)$ against the term $u\left(\theta, z_{R}, t\right) r_{0} d \theta$, and integrating this with respect to $\theta$, gives extracted waveforms of the $n_{E}$ th circumferential mode as

$$
\begin{aligned}
u_{n_{E}}^{\text {ext }}\left(z_{R}, t\right) & =\int_{0}^{2 \pi} u\left(\theta, z_{R}, t\right) \exp \left(-i n_{E} \theta\right) r_{0} d \theta \\
& =2 \pi r_{0} a_{n_{E}} \exp \left(i k_{n_{E}} z_{R}-i \omega t\right) .
\end{aligned}
$$

In practice, receiving sensors are not infinitesimal as indicated by $r_{0} d \theta$, and the number of receiving points is finite. Assuming $N$ receiving positions in the circumferential direction at regular intervals as

$$
\theta_{k}=\frac{2 \pi}{N}(k-1)
$$

and assuming that a displacement is detected in the region with an aperture of $\theta_{0}=2 \pi / N$, then the received signals $\theta$ $=\theta_{k}$ and $z=z_{R}$ are given by

$$
\begin{aligned}
u^{R}\left(\theta_{k}, z_{R}, t\right) & =\int_{\theta_{k}-\theta_{0} / 2}^{\theta_{k}+\theta_{0} / 2} u\left(\theta, z_{R}, t\right) r_{0} d \theta \\
& =r_{0} \sum_{n=-\infty}^{+\infty} \alpha_{n} f_{n}\left(\theta_{0}\right) \exp \left(i n \theta_{k}+i k_{n} z_{R}-i \omega t\right),
\end{aligned}
$$

where

$$
f_{n}\left(\theta_{0}\right)=\left\{\begin{array}{l}
\theta_{0}, \quad \text { for } n=0, \\
\frac{2 \sin \left(n \theta_{0} / 2\right)}{n}, \text { for } n \neq 0 .
\end{array}\right.
$$

Then, multiplying by the weight function $\exp \left(-i n_{E} \theta_{k}\right)$ and summing with respect to $k$ gives, similar to Eq. (4), the extracted waveforms

$$
\begin{aligned}
u_{n_{E}}^{\mathrm{ext}}\left(z_{R}, t\right)= & r_{0} \sum_{k=1}^{N} \sum_{n=-\infty}^{+\infty} \alpha_{n} f_{n}\left(\theta_{0}\right) \\
& \times \exp \left\{i\left(n-n_{E}\right) \theta_{k}+i k_{n} z_{R}-i \omega t\right\} \\
\approx & r_{0} \alpha_{n_{E}} f_{n_{E}}\left(\theta_{0}\right) \exp \left(i k_{n_{E}} z_{R}-i \omega t\right) .
\end{aligned}
$$

It should be noted here that Eq. (8) holds under the condition of $\left|n_{E}\right| \leqslant N / 2-1$, because modes lower than $n_{E}$ are superposed in Eq. (8) for $\left|n_{E}\right| \geqslant N / 2$. In dispersion curves for torsional modes, those modes higher than $n=8$ do not propagate in the frequency regions used in this study $(30-70 \mathrm{kHz})$. Also, for the highly dispersive modes of $n=4-7$, the detected signals become very broad, and only a very small part 


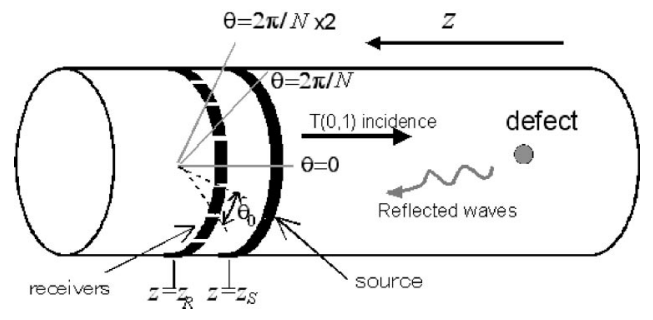

FIG. 2. Schematic setup for defect imaging.

of the energy of these modes can be recorded. Therefore, eight different circumferential positions are used to detect the guided waves in later experiments. This corresponds to $N$ $=8$, and the main propagating modes from $n=-3$ to +3 can be extracted.

\section{B. Waveform prediction of dispersive modes and reconstruction of defect images}

If the diameter, thickness, and material properties of a pipe are given, then the wave numbers $k_{n_{E}}$ and phase velocities $c_{n_{E}}$ of the extracted modes can be theoretically obtained. Once the wave numbers $k_{n_{E}}$ are given, multiplying $\exp \left\{i k_{n_{E}}\left(z-z_{R}\right)\right\}$ against the extracted waveforms $u^{\operatorname{ext}}\left(z_{R}, t\right)$ obtained from received signals at $z=z_{R}$ yields predicted waveforms of the $n_{E}$ th mode at $z=z$ as

$$
\begin{aligned}
& u_{n_{E}}^{\mathrm{ext}}\left(z_{R}, t\right) \exp \left\{i k_{n_{E}}\left(z-z_{R}\right)\right\} \\
& \quad \approx r_{0} \alpha_{n_{E}} f_{n_{E}}\left(\theta_{0}\right) \exp \left(i k_{n_{E}} z-i \omega t\right)=u_{n_{E}}^{\operatorname{ext}}(z, t) .
\end{aligned}
$$

Equation (9) is for a harmonic wave of frequency $\omega$. In actual experiments, extracted waveforms in the frequency domain are obtained by FFT first, and then a series of data multiplied by $\exp \left\{i k_{n_{E}}\left(z-z_{R}\right)\right\}$ are transformed back to the time domain by inverse FFT. This process was presented as a dispersion reduction technique for dispersive Lamb waves by Wilcox et al. ${ }^{14}$

From Eq. (6), waveforms received with uniform weight in the region having an aperture of $\theta_{0}$ at an arbitrary point $(\theta, z)$ on the surface of a pipe are given by

$$
u^{R}(\theta, z, t)=r_{0} \sum_{n=-\infty}^{+\infty} \alpha_{n} f_{n}\left(\theta_{0}\right) \exp \left(i n \theta+i k_{n} z-i \omega t\right) .
$$

Using the predicted extracted waveforms $u_{n}^{\text {ext }}(z, t)$ obtained by Eq. (9), Eq. (10) can be rewritten as

$$
u^{R}(\theta, z, t)=\sum_{n=-\infty}^{n=+\infty} u_{n}^{\operatorname{ext}}(z, t) \exp (\operatorname{in} \theta) .
$$

This equation shows that multiplying $\exp (\operatorname{in} \theta)$ against $u_{n}^{\text {ext }}(z, t)$ obtained in Eq. (9) gives waveforms detected by a receiving transducer with aperture $\theta_{0}$ at an arbitrary point.

The fact that reflected waves from defects are detected at $N$ different circumferential positions (Fig. 2) is now considered. A $\mathrm{T}(0,1)$ mode is emitted at $z=z_{S}$ toward the defects in the $-z$ direction. The $\mathrm{T}(0,1)$ incident wave is reflected back from the defects in the $+z$ direction, and the reflected waveforms are detected by $N$ receiving transducers located at $z$

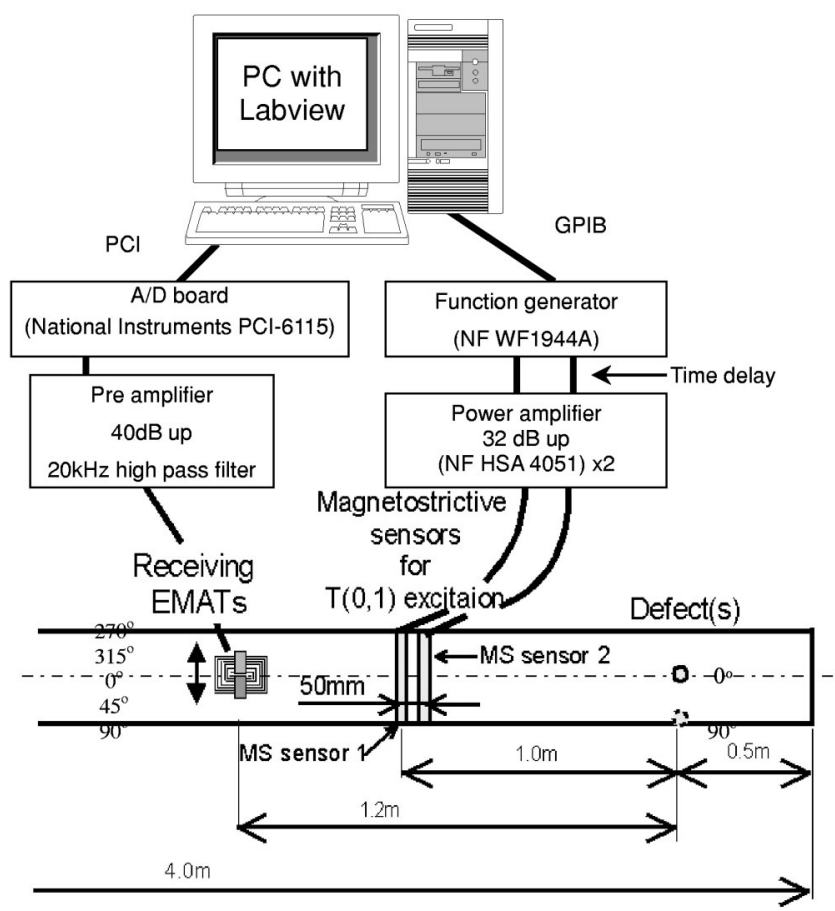

FIG. 3. Experimental setup.

$=z_{R}$. From the $N$ received signals, spatial waveforms at an arbitrary time can be predicted like snapshots at all of the time steps. Since the reflected waveforms are very large at the moment of reflection from a reflective object, the snapshot at this moment shows an image of the reflective object.

\section{EXPERIMENT}

\section{A. Experimental setup}

Figure 3 shows a schematic figure of the experimental setup used in this study. A magnetostrictive sensor (MS sensor) consisting of a nickel plate adhered to the pipe surface and a coil wound on the nickel plate, is used for generating a $\mathrm{T}(0,1)$ mode. ${ }^{15}$ The nickel plates are magnetized in the circumferential direction and serve as bias magnetic fields. Alternating voltage as four-cycle sinusoidal waves of 30, 50, and $70 \mathrm{kHz}$ are applied to the coil, generating vibration in the circumferential direction by magnetostriction. Two magnetostrictive sensors are used to double the $\mathrm{T}(0,1)$ mode signals by applying a time delay to one forward-located sensor (MS sensor2 in Fig. 3). A function generator (NF corporation, WF1944A) and a power amplifier (NF corporation, HSA4051) are used to apply rf signals to the coils.

An electromagnetic acoustic transducer (EMAT) is adopted for noncontact measurements. The experiments of this study require accurate waveform measurements at many different circumferential positions; therefore, noncontact measurement with EMATs is more suitable than contact methods strongly affected by contact pressure and the thickness of the coupling material. The EMAT used in the experiment consists of two permanent magnets (NEOMAX, NEOMAX Co. Ltd., $15 \mathrm{~mm} \times 6 \mathrm{~mm} \times 10 \mathrm{~mm}$ ) and sheet coils fabricated by a printed circuit technique. The EMAT is flexible so that it fits the curvature of the pipe. The sheet coil and 


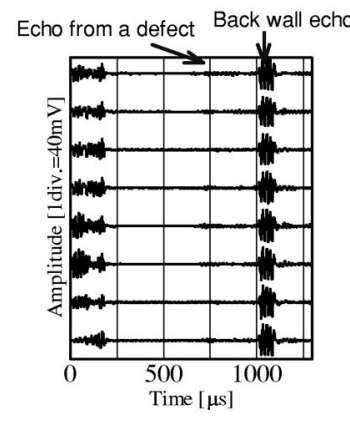

(a) RF signals

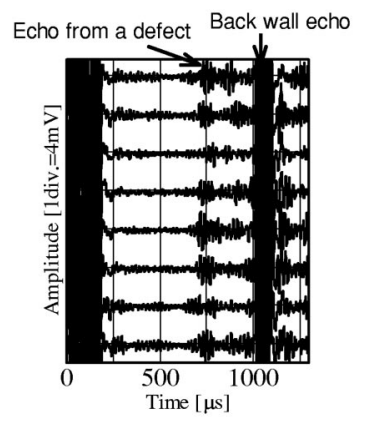

(b) Amplified representation of (a)
FIG. 4. Waveforms detected at eight different circumferential positions. (a) rf signals; (b) amplified representation of (a).

permanent magnets of the EMAT are arranged to receive shear horizontal waves by Lorentz forces. This transducer, then, receives vibration mainly in the circumferential direction. ${ }^{16}$ In order to receive displacement in the region of $1 / 8$ of the circumference, the EMAT is designed to be $44 \mathrm{~mm}$ in width. Signals received at the EMAT are magnified $40 \mathrm{~dB}$ by a preamplifier and are recorded after 10 signal averagings.

The test objects for this study are aluminum pipes of $4 \mathrm{~m}$ in length, $111 \mathrm{~mm}$ in diameter, and $3.5 \mathrm{~mm}$ in thickness with a longitudinal wave velocity $c_{L}=6260 \mathrm{~m} / \mathrm{s}$ and a transverse wave velocity $c_{T}=3080 \mathrm{~m} / \mathrm{s}$. The defects are located $0.5 \mathrm{~m}$ from the pipe end and one meter from MS sensor1 (Fig. 3). Backward waves may propagate in a direction opposite to the defects, but in this study the test pipes are large enough to separate the necessary signals from the unwanted signals that come from the other side of the pipe.

The receiving EMAT is positioned $1.2 \mathrm{~m}$ from the defects and measures signals at eight different circumferential positions. The artificial defects are through-holes of $10 \mathrm{~mm}$ in diameter and are located at $0^{\circ}$ for the one-hole test pipe and at $0^{\circ}$ and $90^{\circ}$ for the two-hole test pipe, in the circumferential direction, as shown in Fig. 3.

Figures 4(a) and 4(b) are the waveforms detected at eight different circumferential positions $\left(0^{\circ}, 45^{\circ}, 90^{\circ}, 135^{\circ}\right.$, $180^{\circ}, 225^{\circ}, 270^{\circ}$, and $315^{\circ}$ ) for a one-hole test pipe and 50 $\mathrm{kHz}$ incidence. Large signals around $1000 \mu$ s are backwall echoes from the pipe end 1.5 and $1.7 \mathrm{~m}$ in distance from excitation MS sensor1 and the receiving EMAT, respectively. Small signals between 500 and $1000 \mu$ s are echoes from the defect. Figure 4(b) shows the waveforms of Fig. 4(a) after amplification to discern the waveforms of the defect echoes. The backwall echoes and incident waves around $t=0 \mu \mathrm{s}$ are unnecessary for defect imaging, so all signals from 330 to $980 \mu$ s are extracted, and other amplitudes are assumed to be zero.

\section{B. Prediction of waveforms reflected at a pipe defect}

Figure 5 shows spatial waveforms at three different times predicted by the above process using the detected waveforms of Fig. 4. All images show absolute values of spatial waveforms normalized by the maximum amplitude of spatial waveforms at all time steps. Since (a) $t=195 \mu \mathrm{s}$ is a time when the incident $\mathrm{T}(0,1)$ mode has not yet arrived at the

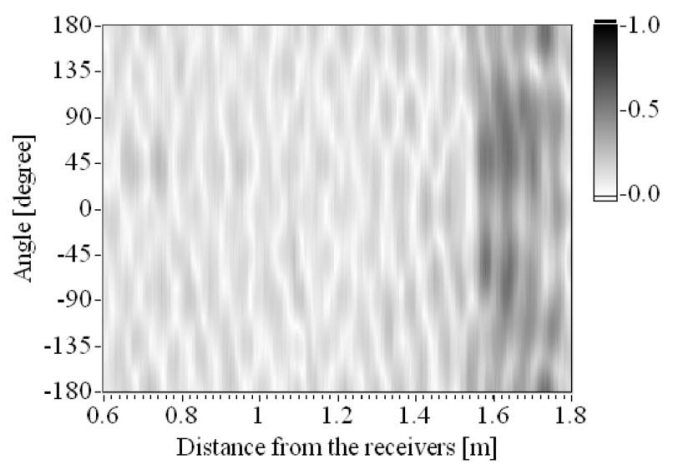

(a) $t=195 \mu \mathrm{s}$

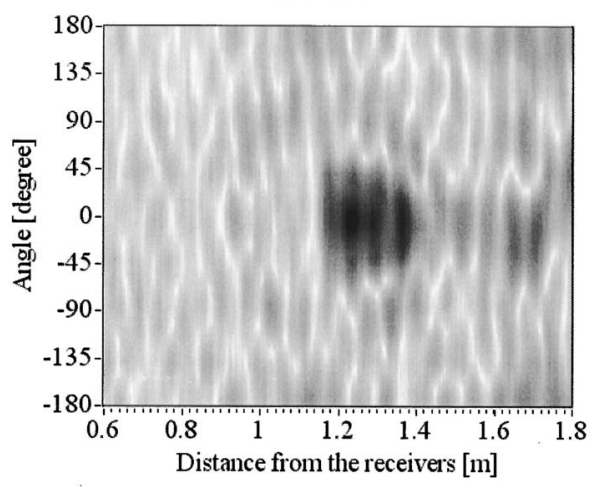

(b) $t=325 \mu \mathrm{s}$

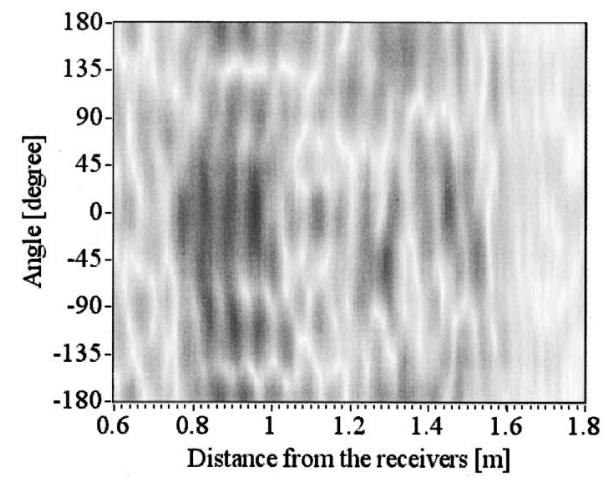

(c) $t=455 \mu \mathrm{s}$

FIG. 5. Snapshots of predicted waveforms at various moments. (a) $t$ $=195 \mu \mathrm{s}$, (b) $t=325 \mu \mathrm{s}$, (c) $t=455 \mu \mathrm{s}$.

defect, the predicted waveform (a) does not give a defect image. In (b), the moment when the incident $\mathrm{T}(0,1)$ mode arrives at the defect, a distinct spot with high intensity is present at approximately $1.2 \mathrm{~m}$ from the receivers and $0^{\circ}$, which agrees well with the position of the artificial defect. After (b), the waveforms spread out from the defect, as can be seen in (c).

\section{Effect of frequency on the predicted waveforms}

Next, snapshots of the predicted waveforms in the onehole pipe at $t=325 \mu \mathrm{s}$ are shown in Fig. 6 for a four-cycle sinusoidal incidence of 30 and $70 \mathrm{kHz}$. For (a) $30 \mathrm{kHz}$, the high-intensity spot becomes broad in both the longitudinal and circumferential directions, indicating lower resolution. On the other hand, a better result with higher resolution is obtained in both directions for (b) $70 \mathrm{kHz}$.

Since resolution in the longitudinal direction is determined by a wave disturbance zone, then in the case of four- 


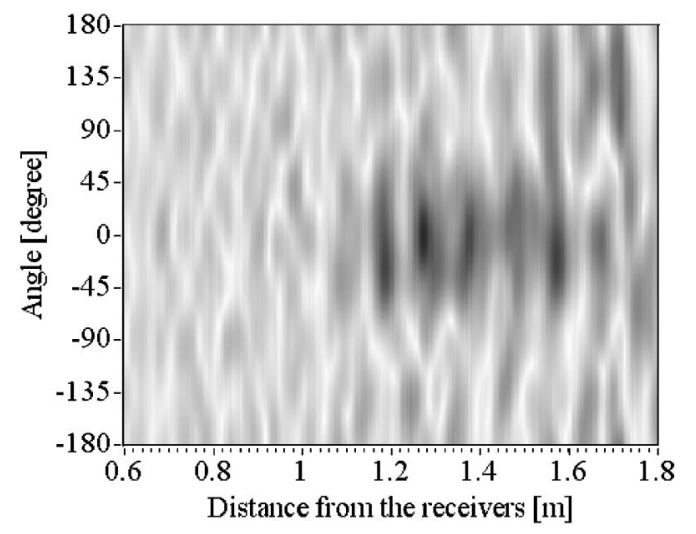

(a) $30 \mathrm{kHz}, t=325 \mu \mathrm{s}$

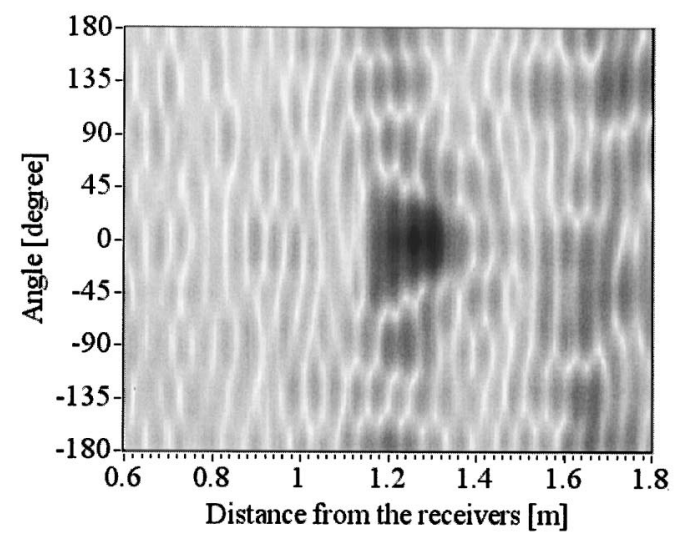

(b) $70 \mathrm{kHz}, t=325 \mu \mathrm{s}$

FIG. 6. Predicted waveforms for various frequencies at $t=325 \mu \mathrm{s}$. (a) 30 $\mathrm{kHz}, t=325 \mu \mathrm{s}$; (b) $70 \mathrm{kHz}, t=325 \mu \mathrm{s}$.

cycle incidence, four times the wavelength is taken as the high-intensity zone. Circumferential resolution is determined by the order of detectable circumferential modes. In the frequency region of approximately $30 \mathrm{kHz}$, modes of orders higher than $\mathrm{T}(3,1)$ cannot be detected due to the cutoff frequency, and the $\mathrm{T}(2,1)$ and $\mathrm{T}(3,1)$ modes have large dispersion. It is expected, therefore, that signals within the given gate $(t=330$ to $980 \mu \mathrm{s})$ do not contain all of the energy of the $\mathrm{T}(2,1)$ and $\mathrm{T}(3,1)$ modes, causing low resolution in the circumferential direction. In contrast, in the high-frequency region of approximately $70 \mathrm{kHz}$, since higher-order modes such as $\mathrm{T}(2,1)$ and $\mathrm{T}(3,1)$ have low-dispersion characteristics, most of the energy of the higher-order modes can be obtained, resulting in higher resolution in the circumferential direction.

Figure 7 shows snapshots at the moment when the incident $\mathrm{T}(0,1)$ mode arrives at the defects $(t=325 \mu \mathrm{s})$ for a two-hole test pipe with through-holes at $0^{\circ}$ and $90^{\circ}$ in the three different frequency regions. In the 30 and $50 \mathrm{kHz}$ defect images, single broad dark regions can be seen, the centers of which are located at about $45^{\circ}$. In the $70 \mathrm{kHz}$ defect image, however, two distinct dark spots can be seen at $0^{\circ}$ and $90^{\circ}$, at $1.2 \mathrm{~m}$.

The dark zones even at $70 \mathrm{kHz}$ for both one-hole and two-hole test pipes are shown to be larger than the artificial holes of $10 \mathrm{~mm}$ in diameter. We can expect that the dark

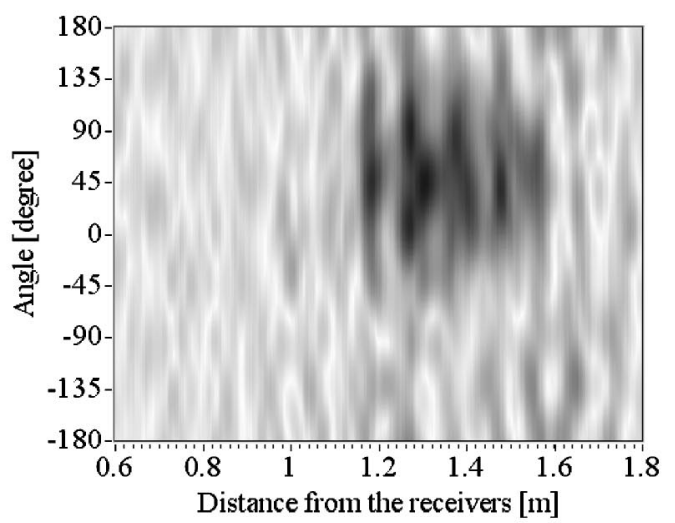

(a) $30 \mathrm{kHz}$

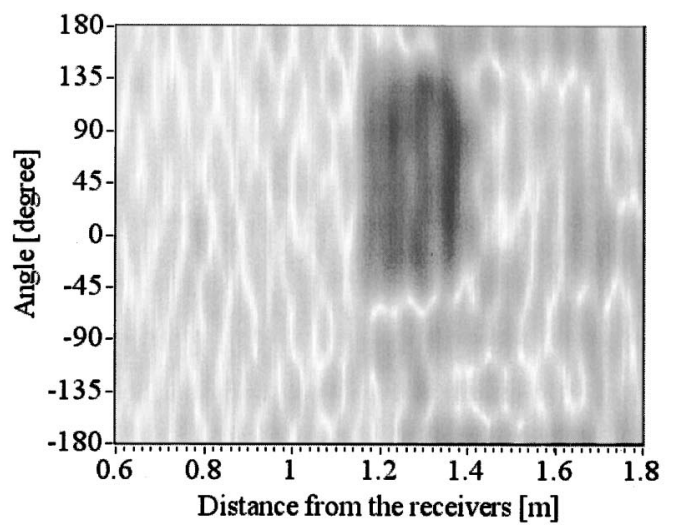

(b) $50 \mathrm{kHz}$

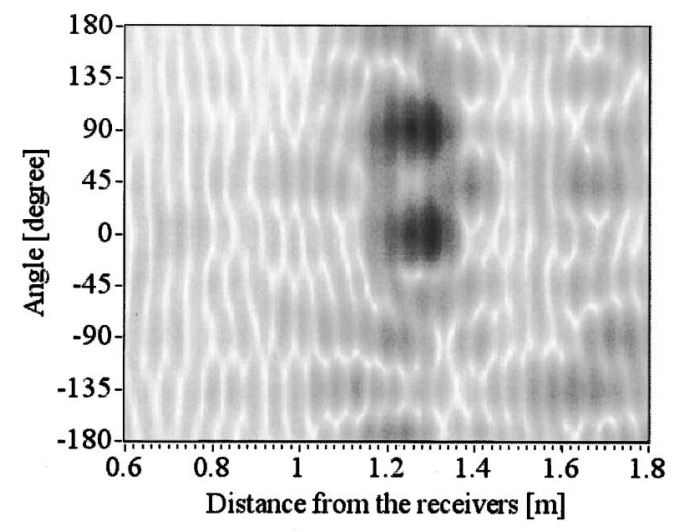

(c) $70 \mathrm{kHz}$

FIG. 7. Predicted waveforms at $t=325 \mu \mathrm{s}$ for a two-hole test pipe and various frequencies. (a) $30 \mathrm{kHz}$, (b) $50 \mathrm{kHz}$, (c) $70 \mathrm{kHz}$.

zones become closer to the circle $10 \mathrm{~mm}$ in diameter at higher frequencies. However, since the predicted waveform, as shown in Eq. (10), denotes the waveform at $(\theta, z, t)$ measured by the receiving transducer with an aperture of $\theta_{0}$ $=2 \pi / N$, the resolution in the circumferential direction is theoretically limited to the aperture of receiving transducers $\theta_{0}=2 \pi / N$.

\section{Defect imaging considering the arrival region of the incident wave}

In Figs. 5-7, which show the waveforms predicted from the received signals, the defect images are obtained as snapshots at the moment that the incident $\mathrm{T}(0,1)$ mode arrives at 


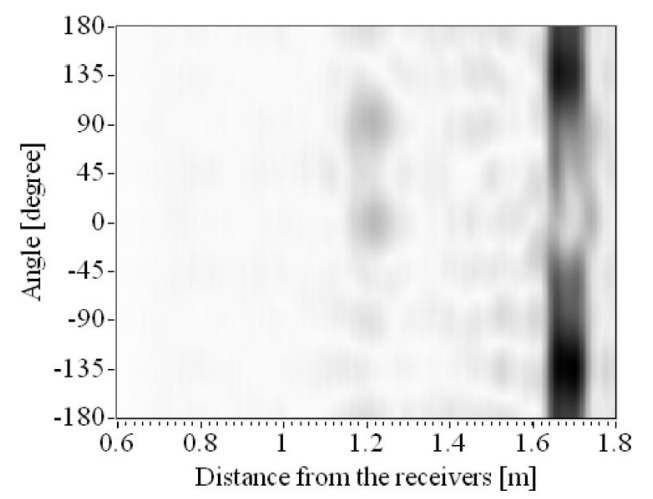

FIG. 8. Imaging of defects and a pipe end.

a defect region. However, when reflection objects are located at different longitudinal locations, information on the reflection objects in the longitudinal direction cannot be obtained in one snapshot. In this section we describe defect imaging in which such reflection objects are shown in one image. Summing up the images of predicted waveforms at all time steps only in the arrival region of incident wave $\mathrm{T}(0,1)$ provides an image of reflection objects, such as defects, pipe ends, weld lines, and branches. Acquiring an image of the two holes and pipe end using the received signals that produced the snapshot image of Fig. 7(c) is now considered. The image in Fig. 7(c) was obtained by gating the received signals between 330 and $980 \mu \mathrm{s}$ in order to avoid backwall echoes. In the following process, however, the signals are gated between 330 and $1200 \mu \mathrm{s}$ in order to include reflection waves from the pipe end.

Now, consider the arrival region of an incident $\mathrm{T}(0,1)$ mode at a certain time $t$, which is from $z=c_{T} t-l \lambda$ to $z$ $=c_{T} t$, where $l$ and $\lambda$ are the number of cycles and the wavelength of the incident burst waves, respectively. If reflective walls, such as defects, exist in this region, then the predicted waveforms of the reflected waves become large. In contrast, if there are no reflective objects present, then the predicted waveforms become zero. Thus, summing up the absolute values of the predicted waveforms in the regions for all time steps gives an image of the reflective objects. This is shown in Fig. 8, in which a reflective object with high intensity is present at approximately $1.7 \mathrm{~m}$ from the receivers, and two round reflective objects with low intensity are present at 1.2 $\mathrm{m}, \theta=0^{\circ}$ and $\theta=90^{\circ}$. The two dark regions at $1.2 \mathrm{~m}$ agree well with the two artificial holes. The dark region at $1.7 \mathrm{~m}$ should indicate the pipe ends, but is not symmetric with respect to the pipe axis and has a nonreflecting region behind the two holes. It is considered that this region cannot be reconstructed as an image due to scattering at the holes and multiple reflections between the holes and the pipe end. However, it is confirmed that this imaging technique has a potential for providing images of reflective objects having longitudinal distributions.

\section{E. Possible errors by measurement conditions}

The imaging technique using guided waves presented herein is applicable when guided wave modes can be perfectly separated and dispersion curves are theoretically ob- tained. However, it is possible to superpose unwanted modes and to change a defect image due to the characteristic of the receivers. In this study, we assume that the EMATs detect only horizontal shear vibration by Lorentz force, but the EMATs can also detect unexpected longitudinal or out-ofplane vibrations. In contrast, if we can separately detect vibrations in all directions and extract flexural modes as well as torsional modes, better defect images can be obtained.

Random noise, largely detected as shown in Fig. 4, may also distort defect images. However, random noise interfering with received signals is reduced in the summation with respect to $k$ in Eq. (8). Therefore, the dark defect zone in Fig. 5(b) appears large compared with other noisy regions. This indicates the robustness of the defect imaging technique against random noise interfering with each waveform.

In this technique, however, the sensitivity of the transducers at all receiving points should coincide because all signals are added and subtracted in the imaging process. Since we use one EMAT for receiving at eight positions in this study, this error must be small, but when using the contact technique, in which it is difficult to control contact pressure and the thickness of the coupling medium, sensitivity mismatching could result in large distortion in defect images. In order to investigate the influence of differences in sensor sensitivity, Fig. 9 shows the defect images when specific signals cannot be properly obtained, for a two-hole test pipe and $70 \mathrm{kHz}$ incidence. Signals were gated from 330 to $980 \mu \mathrm{s}$ in order to obtain only images of defects and to avoid pipe ends. The defect image in Fig. 9(a) was constructed by using all of the signals, the same signals that were used to obtain Figs. 7(c) and 8. Figures 9(b) and 9(c) were obtained by replacing the first signals received at $\theta=0^{\circ}$ and the first two signals at $\theta=0^{\circ}, 45^{\circ}$ with zero-valued samples, respectively, showing the typical case when a portion of the signals cannot be detected due to transducer trouble in practical testing. In Fig. 9(d), only the first signals at $\theta=0^{\circ}$ are phase-reversed and the other signals are the same, indicating an insufficient contact condition for the contact-type transducers, or a difference in sensor sensitivity. Comparing Figs. 9(a), 9(b), and 9(c), as the number of zero signals increases, a more unwanted dark zone can be seen in the images. In Fig. 9(d), a very large distortion of the defect images can be seen, although only one waveform was reversed. These results reveal that differences in transducer sensitivity, as well as a lack of signals, may cause large image distortion.

\section{CONCLUSIONS}

Defect imaging with guided waves was described. A defect image in a pipe was obtained as a spatial waveform by processing signals detected at many different circumferential positions. After a detailed theoretical description, the defect imaging technique was experimentally verified using eight signals detected at eight different circumferential points.

In defect imaging tests for 30,50 , and $70 \mathrm{kHz}$ four-cycle sinusoidal waves, higher resolutions in both the longitudinal and circumferential directions were obtained at higher frequencies. Reconstructed images for two-hole and one-hole pipes agreed well with the test pipes. Moreover, a pipe end 

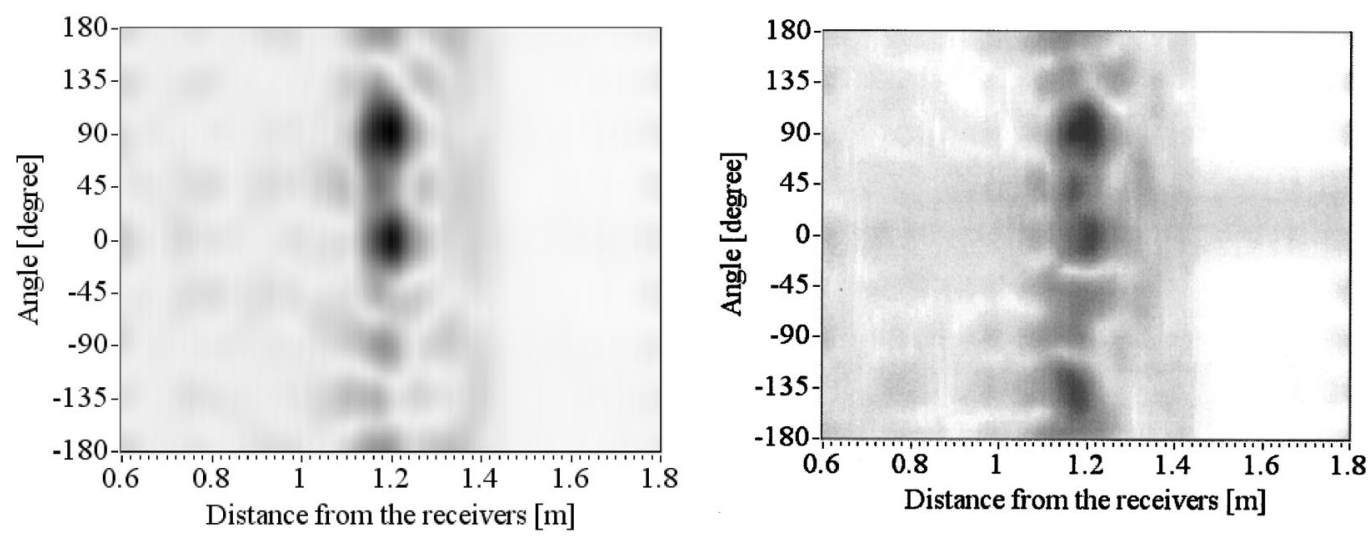

(a) All signals considered

(c) The first two signals received at $\theta=0^{\circ}$ and $\theta=45^{\circ}$ are replaced with zero-valued samples

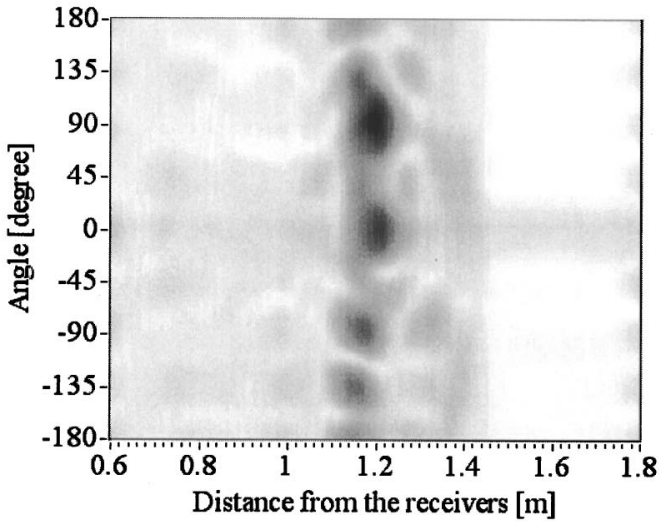

(b) The first signal received at $\theta=0^{\circ}$ are replaced with zero-valued samples

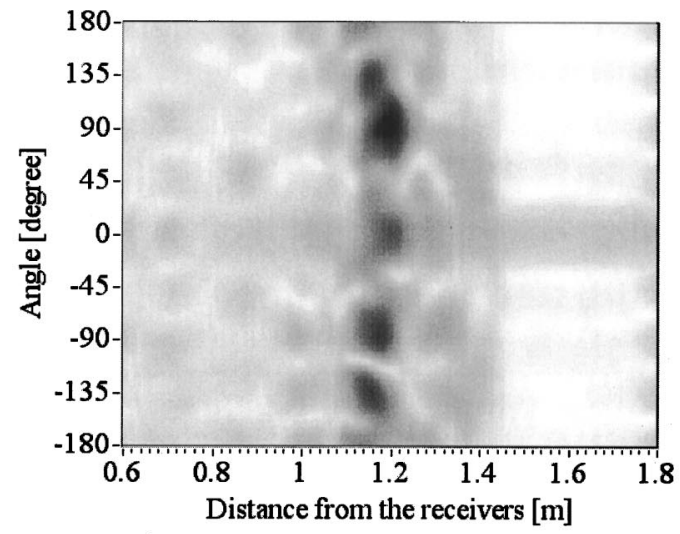

(d) The first signals at $\theta=0^{\circ}$ are phase-reversed.

FIG. 9. Defect images when specific signals cannot be properly obtained, for a two-hole test pipe and $70 \mathrm{kHz}$ incidence. (a) All signals considered. (b) The first signals received at $\theta=0^{\circ}$ are replaced with zero-valued samples. (c) The first two signals received at $\theta=0^{\circ}$ and $\theta=45^{\circ}$ are replaced with zero-valued samples. (d) The first signals at $\theta=0^{\circ}$ are phase-reversed.

behind two holes could be seen in the image, but an undetectable region was found just behind the holes.

In this study, we used only torsional modes and shear horizontal vibration. From a theoretical point of view, the imaging technique described herein can be applied to longitudinal and flexural modes. Because flexural modes in reflected waves from defects were detectable, combining this technique with other modes should enhance the defect images.

\section{ACKNOWLEDGMENTS}

This research was supported by the Industrial Technology Research Grant Program in 2003 from the New Energy and Industrial Technology Development Organization (NEDO) of Japan. We thank Takashi Ikeda at the CXR Corporation for his considerable assistance with the magnetostrictive sensors.

${ }^{1} \mathrm{~J}$. L. Rose, "A baseline and vision of ultrasonic guided wave inspection potential," J. Pressure Vessel Technol. 124, 273-282 (2002).

${ }^{2}$ P. Cawley, M. J. S. Lowe, D. N. Alleyne, B. Pavlakovic, and P. Wilcox, "Practical long range guided wave inspection-applications to pipes and rail," Mater. Eval. 61, 67-74 (2003).

${ }^{3}$ R. K. Ing and M. Fink, “Time reversed Lamb waves," IEEE Trans. Ultrason. Ferroelectr. Freq. Control 45, 1032-1043 (1998).

${ }^{4}$ T. Hayashi and J. L. Rose, "Guided wave simulation and visualization by a semi-analytical finite element method," Mater. Eval. 61, 75-79 (2003).

${ }^{5}$ J. J. Ditri, "Utilization of guided elastic waves for the characterization of circumferential crack in hollow cylinders," J. Acoust. Soc. Am. 96, 37693775 (1994)

${ }^{6}$ M. J. S. Lowe, D. N. Alleyne, and P. Cawley, "The mode conversion of a guided wave by a part-circumferential notch in a pipe," J. Appl. Mech. 65, 649-656 (1998)

${ }^{7}$ D. N. Alleyne, M. J. S. Lowe, and P. Cawley, "The reflection of guided waves from circumferential notches in pipes," J. Appl. Mech. 65, 635641 (1998).

${ }^{8} \mathrm{~J}$. Li and J. L. Rose, "Excitation and propagation of non-axisymmetric guided waves in a hollow cylinder," J. Acoust. Soc. Am. 109, 457-468 (2001).

${ }^{9}$ A. Demma, P. Cawley, M. Lowe, and A. G. Roosenbrand, "The reflection of the fundamental torsional mode from cracks and notches in pipes," J. Acoust. Soc. Am. 114, 611-625 (2003).

${ }^{10}$ D. C. Gazis, "Three-dimensional investigation of the propagation of waves in hollow circular cylinders. I. Analytical foundation. II. Numerical Results," J. Acoust. Soc. Am. 31, 568-578 (1959).

${ }^{11}$ J. L. Rose, Ultrasonic Waves in Solid Media (Cambridge University Press, Cambridge, 1999).

${ }^{12}$ T. Hayashi, K. Kawashima, Z. Sun, and J. Rose, "Analysis of flexural mode focusing by a semi-analytical finite element method," J. Acoust. Soc. Am. 113, 1241-1248 (2003).

${ }^{13}$ H. Nishino, S. Takashina, F. Uchida, M. Takemoto, and K. Ono, "Modal analysis of hollow cylindrical guided waves and applications," Jpn. J. Appl. Phys. 40, 364-370 (2001).

${ }^{14}$ P. D. Wilcox, M. J. S. Lowe, and P. Cawley, "A signal processing technique to remove the effect of dispersion from guided wave signals," Rev. Prog. Quant. Nondestr. Eval. 20, 555-562 (2001).

${ }^{15}$ H. Kwun, S. Y. Kim, and G. M. Light, "The magnetostrictive sensor technology for long range guided wave testing and monitoring of structures," Mater. Eval. 61, 80-84 (2003).

${ }^{16}$ M. Hirao and H. Ogi, EMATs For Science and Industry—Noncontacting Ultrasonic Measurements (Kluwer, Dordrecht, 2003). 